・研究简报・

\title{
6,7-二甲氧基-4-哌嗪喹唑啉缩氨基硫腿衍生物的合成及 体外抗肿瘤活性研究
}

\author{
刘海涁* 徐惠娟吕萍潘宁宁李双奇 \\ (辽宁科技学院生物医药与化学工程学院 本溪 117004)
}

\begin{abstract}
摘要 以 4,5-二甲氧基-2-氨基苯甲酸和醋酸甲眯为起始原料, 经环化、氯化、取代和缩合四步反应，设计、合成了六 个未见文献报道的含有缩氨基硫腿的喹唑啉衍生物 $\mathbf{4 a} \sim \mathbf{4 f}$, 其结构用 ${ }^{1} \mathrm{H} \mathrm{NMR},{ }^{13} \mathrm{C}$ NMR, ESI-MS, IR 及元素分析测试 技术进行了表征. 采用 MTT 法测试了化合物 $4 \mathbf{a} \sim \mathbf{4 f}$ 对人胃癌 SGC-7901、人口腔表皮样癌 KB 和人纤维肉瘤 HT- 1080 的体外抗肿瘤活性. 初步的测试结果表明，化合物 $4 \mathbf{a}$ 和 $4 \mathbf{f}$ 对 HT1080 表现出显著的抗肿瘤活性.

关键词＼cjkstart喹唑啉; 缩氨基硫嫝; 抗肿瘤活性; MTT 法
\end{abstract}

\section{Synthesis and in vitro Antitumor Activities of 6,7-Dimethoxy-4-piperazinquinazoline Thiosemicarbazone Derivatives}

\author{
Liu, Haibin* $\quad$ Xu, Huijuan $\quad$ Lü, Ping $\quad$ Pan, Ningning $\quad$ Li, Shuangqi \\ (School of Biomedical \& Chemical Engineering, Liaoning Institute of Science and Technology, Benxi 117004)
}

\begin{abstract}
Six new quinazoline derivatives $\mathbf{4 a} \sim \mathbf{4} \mathbf{f}$ bearing thiosemicabazone have been designed and synthesized by four step reactions of cyclization, chlorination, substitution and condensation using 2-amino-4,5-dimethoxybenzoic acid and methanimidamide acetate as starting materials. Their structures were confirmed by ${ }^{1} \mathrm{H}$ NMR, ${ }^{13} \mathrm{C}$ NMR, ESI-MS, IR techniques and elemental analysis. The in vitro antitumor activities of compounds $\mathbf{4 a} \sim \mathbf{4} \mathbf{f}$ against SGC-7901 (human gastric cancer), KB (human oral epidermoid cancer) and HT-1080 (human fibrosarcoma) cell lines were tested using colorimetric MTT assay. The preliminary results indicated that compounds $\mathbf{4 a}$ and $\mathbf{4 f}$ showed significant antitumor activity against HT1080.
\end{abstract}

Keywords quinazoline; thiosemicarbazone; antitumor activity; MTT assay

6,7-二甲氧基喹唑啉类化合物具有优良的抗肿瘤活 性，许多化合物通过抑制 EGFR(表皮生长因子受体)、 FGFR(成纤维细胞生长因子受体)、AR(肾上腺素能受 体)、PDGFR(血小板衍生生长因子受体)或 VEGF(血管 内皮生长因子)等而表现出抗前列腺癌、抗肺癌、抗乳 腺癌等作用 ${ }^{[1 \sim 5]}$. 因此, 有关该类化合物的分子设计、合
成及生物活性的研究是当前新药创制的热点之一[6 8]. CT52923 是一种 4-哌嗪取代的喹唑啉类化合物(图 1), 测得其对 NIH/3T3 细胞系的 PDGF 抑制率大多可达 $80 \%$ 以上. 同时发现, CT52923 与 PDGF-A, PDGF-B 和 PDGF-C, PDGF-D 分别有不同的配体作用, 能抑制多种 癌细胞, 而对正常细胞没有损伤 ${ }^{[4]}$.

\footnotetext{
*E-mail: haibin0616@163.com

Received August 17, 2011; revised November 28, 2011; accepted November 29, 2011.
}

Project was supported by the Program of Liaoning Provincial Committee of Education (No. L2010204), the Program for Liaoning Excellent Talents in University (No. LJQ2011130) and the Ph. D. Program Foundation of Liaoning Institute of Science and Technology (No. 0906B1).

辽宁省教育厅科研项目(No. L2010204)、辽宁省高等学校优秀人才支持计划(No. LJQ2011130)、辽宁科技学院博士启动金(No. 0906B1)资助项目. 
<smiles>COc1cc2ncnc(N3CCN(C(=S)NCc4ccc5c(c4)OCO5)CC3)c2cc1OC</smiles>

图 1 CT52923 的结构式

Figure 1 The structure of CT52923

缩氨基硫嫝及其衍生物由于具有广泛的生物活性, 如抗病毒、抗菌、抗肿瘤、抗结核、抗疮等而备受人们 的重视 ${ }^{[9 \sim 13]}$. 在缩氨基硫嫝化合物中, $\mathrm{C}=\mathrm{N}^{1} \mathrm{NH}$ $\mathrm{HC}(\mathrm{S}) \mathrm{N}^{4}$ 结构是缩氨基硫脲化合物具有生物活性的基本 活性单元，N(1)上醛酮的不同结构及 N(4)上不同结构的 活性基团, 对其抗菌、抗肿瘤等多种生物活性的强弱将 产生极大影响 ${ }^{[14]}$.

为了寻找具有高效抗肿瘤活性的药物, 本工作以 CT52923 为先导化合物, 用 4,5-二甲氧基-2-氨基苯甲酸 经闭环、氯化、取代和缩合反应设计并合成了 6 个含哌 嗪的喹唑啉缩氨基硫脲类新化合物(Scheme 1). 通过 IR, ${ }^{1} \mathrm{H} N M R,{ }^{13} \mathrm{C}$ NMR 和 MS 技术对其结构进行了表征, 并 进一步采用 MTT 法对所合成的 6 个新化合物进行了对 人胃癌 SGC-7901、人口腔表皮样癌 KB 和人纤维肉瘤 HT-1080 的体外抗肿瘤活性测试, 结果表明: 化合物 4a 和 $4 f$ 对 $\mathrm{HT}-1080$ 细胞的 $\mathrm{IC}_{50}$ 值为 $10.43 \mu \mathrm{mol} / \mathrm{L}$ 和 28.57 $\mu \mathrm{mol} / \mathrm{L}$.

\section{1 实验部分}

\section{1 主要仪器与试剂}

WRS-1B 数字熔点仪(温度计未经校正); Bruker avance 500 型核磁共振仪(TMS 为内标); Thermo LCQ Fleet HPLC-MS 质谱仪; PE Spectrum 100 红外光谱仪; Vario EL III 元素分析仪; JM5102 型电子天平; RE52-99 旋转蒸发仪; ZF-I 三用紫外分析仪. 肼基二硫代甲酸甲 酯按文献[15]方法制备；4,5-二甲氧基-2-氨基苯甲酸和 醋酸甲眯从上海嘉辰化工有限公司购买; 所用试剂和溶 剂均为分析纯.

\subsection{6,7-二甲氧基喹唑啉-4(3H)-酮(1)的制备}

向连有回流冷凝装置的 $250 \mathrm{~mL}$ 圆底烧瓶中加入 4,5-二甲氧基-2-氨基苯甲酸 $(7.88 \mathrm{~g}, 0.04 \mathrm{~mol}$ )和醋酸甲 脒 $(8.32 \mathrm{~g}, 0.08 \mathrm{~mol})$, 再加入 $100 \mathrm{~mL}$ 无水乙醇, 回流 10 $\mathrm{h}$, 然后冷却至室温, 用减压蒸除溶剂, 加入 $50 \mathrm{~mL}$ 饱和 碳酸氢钠溶液调节 $\mathrm{pH}$ 在 7 8 之间，过滤，滤饼用少量 水淋洗，得到灰色粉末 $7.12 \mathrm{~g}$ ，产率 86.4\%， m.p.>300 ${ }^{\circ} \mathrm{C} ;{ }^{1} \mathrm{H}$ NMR (DMSO- $\left.d_{6}, 500 \mathrm{MHz}\right) \delta: 7.97(\mathrm{~s}, 1 \mathrm{H}), 7.43$ $(\mathrm{s}, 1 \mathrm{H}), 7.12(\mathrm{~s}, 1 \mathrm{H}), 3.89\left(\mathrm{~s}, 3 \mathrm{H}, \mathrm{OCH}_{3}\right), 3.85(\mathrm{~s}, 3 \mathrm{H}$, $\left.\mathrm{OCH}_{3}\right)$.
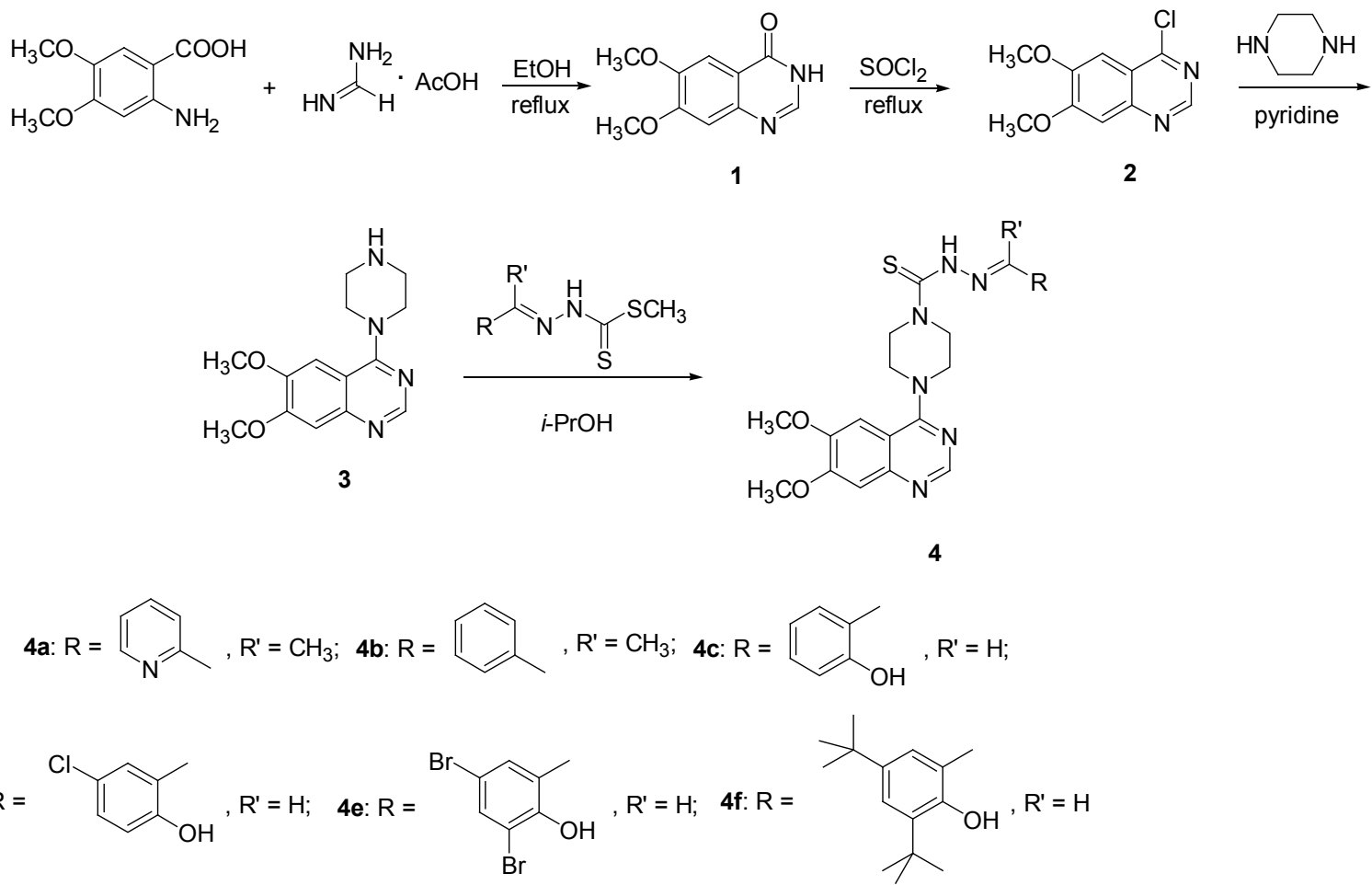

图式 1 6,7-二甲氧基-4-哌溙喹唑啉缩氨基硫腿衍生物的合成

Scheme 1 Synthesis of 6,7-dimethoxy-4-piperazinquinazoline thiosemicarbazone derivatives 


\subsection{4-氯-6,7-二甲氧基喹唑啉(2)的制备}

将 6,7-二甲氧基喹唑啉-4-酮 $(1,6.18 \mathrm{~g}, 0.03 \mathrm{~mol})$ 加 入到连有回流冷凝装置和尾气吸收装置的 $250 \mathrm{~mL}$ 的圆 底烧瓶中, 再加入 $160 \mathrm{~mL}$ 二氯亚砜, 回流反应约 $8 \mathrm{~h}$, 至无氯化氢气体放出为止. 减压蒸除二氯亚砜, 将所得 固体物质慢慢加到 $100 \mathrm{~g}$ 碎冰中, 用玻璃棒充分搅拌, 稀氨水调节其 $\mathrm{pH}$ 值在 7 左右, 过滤, 干燥, 得到 $6.01 \mathrm{~g}$ 浅黄色固体，产率 $89.4 \%$, m.p. $182.2 \sim 183.1{ }^{\circ} \mathrm{C} ;{ }^{1} \mathrm{H}$ NMR (DMSO- $\left.d_{6}, 500 \mathrm{MHz}\right) \delta: 8.94(\mathrm{~s}, 1 \mathrm{H}), 7.46(\mathrm{~s}, 1 \mathrm{H})$, $7.35(\mathrm{~s}, 1 \mathrm{H}), 3.91\left(\mathrm{~s}, 3 \mathrm{H}, \mathrm{OCH}_{3}\right), 3.89\left(\mathrm{~s}, 3 \mathrm{H}, \mathrm{OCH}_{3}\right)$.

\subsection{6,7-二甲氧基-4-(1-哌嗪基)喹唑啉(3)的制备}

向连有回流冷凝装置的 $250 \mathrm{~mL}$ 圆底烧瓶中加入哌 嗪(12.04 g, $0.14 \mathrm{~mol})$ 和 $90 \mathrm{~mL}$ 吡啶, 搅拌至哌嗪完全溶 解后加入 4-氯-6,7-二甲氧基喹唑啉 $(2,11.2 \mathrm{~g}, 0.05 \mathrm{~mol})$, 于 $135{ }^{\circ} \mathrm{C}$ 油浴中进行反应, 回流 $8 \mathrm{~h}$, 冷却至室温, 减压 蒸发除去溶剂, 粗产品经柱层析分离提纯, 洗脱液为二 氯甲烷/甲醇 $(V: V=15: 1)$, 得到黄色固体 $10.31 \mathrm{~g}$, 产 率 75.3\%, m.p. $156.3 \sim 159.2{ }^{\circ} \mathrm{C} ;{ }^{1} \mathrm{H}$ NMR $\left(\mathrm{CDCl}_{3}, 500\right.$ MHz) $\delta: 8.67$ (s, 1H), 7.25 (s, 1H), $7.11(\mathrm{~s}, 1 \mathrm{H}), 4.03$ (s, $\left.3 \mathrm{H}, \mathrm{OCH}_{3}\right), 3.99\left(\mathrm{~s}, 3 \mathrm{H}, \mathrm{OCH}_{3}\right), 3.64(\mathrm{t}, J=5 \mathrm{~Hz}, 4 \mathrm{H}$, $\left.\mathrm{CH}_{2}\right), 310$ (t, $\left.J=5 \mathrm{~Hz}, 4 \mathrm{H}, \mathrm{CH}_{2}\right) ; \mathrm{MS}\left(\mathrm{APCI}^{+}\right) \mathrm{m} / z(\%)$ : $275.01([\mathrm{M}+1], 100)$.

\subsection{6,7-二甲氧基-4-哌嗪喹唑啉缩氨基硫脲化合物 $4 \mathrm{a} \sim 4 \mathrm{f}$ 的制备}

向连有尾气吸收装置的 Schlenk 反应器中加入肼基 二硫代甲酸甲酯席夫碱(0.001 mol)和 $5 \mathrm{~mL}$ 异丙醇, 加 热至完全溶解后再加入 6,7-二甲氧基-4-(1-哌嗪基)喹唑 啉 $(3,0.274 \mathrm{~g}, 0.001 \mathrm{~mol})$, 回流 $24 \mathrm{~h}$, 甲硫醇尾气用乙醇 吸收, 冷却至室温后, 生成的黄色沉淀过滤, 自然干燥, 经柱层析分离, 洗脱液为二氯甲烷: 甲醇 $(V: V=15$ : 1 ), 得到目标产物 6,7-二甲氧基-4-哌嗪基喹唑啉缩氨基 硫脲化合物 $\mathbf{4 a} \sim \mathbf{4 f}$.

4a: 橙黄色固体, 51.6\%, m.p. $234.9 \sim 235.7{ }^{\circ} \mathrm{C}$; $(Z$ 式和 $E$ 式, $Z: E \approx 1: 1){ }^{1} \mathrm{H}$ NMR (DMSO- $d_{6}, 500 \mathrm{MHz}$ ) $\delta: 14.83(\mathrm{~s}, 1 \mathrm{H}), 10.04(\mathrm{~s}, 1 \mathrm{H}), 8.77(\mathrm{~d}, J=4.5 \mathrm{~Hz}, 1 \mathrm{H})$, $8.60 \sim 8.56(\mathrm{~m}, 3 \mathrm{H}), 8.04(\mathrm{t}, J=8.5 \mathrm{~Hz}, 1 \mathrm{H}), 7.97$ (d, $J=$ $4.5 \mathrm{~Hz}, 1 \mathrm{H}), 7.92$ (d, $J=8 \mathrm{~Hz}, 1 \mathrm{H}), 7.85 \sim 7.79$ (m, 1H), $7.57(\mathrm{t}, J=5 \mathrm{~Hz}, 1 \mathrm{H}), 7.38(\mathrm{dd}, J=5.5,6 \mathrm{~Hz}, 1 \mathrm{H}), 7.25 \sim$ $7.23(\mathrm{~m}, 3 \mathrm{H}), 5.77(\mathrm{~s}, 1 \mathrm{H}), 4.43$ (t, $J=5 \mathrm{~Hz}, 4 \mathrm{H}, 2-\mathrm{CH}_{2}$, 哌嗪环), 3.94 (t, $J=5 \mathrm{~Hz}, 4 \mathrm{H}, 2-\mathrm{CH}_{2}$, 哌嗪环), 4.26 (t, $J=5 \mathrm{~Hz}, 4 \mathrm{H}, 2-\mathrm{CH}_{2}$, 哌嗪环), $3.75(\mathrm{t}, J=5 \mathrm{~Hz}, 4 \mathrm{H}$, 2- $\mathrm{CH}_{2}$, 哌嗪环), $3.96\left(\mathrm{~s}, 3 \mathrm{H}, \mathrm{OCH}_{3}\right), 3.94\left(\mathrm{~s}, 3 \mathrm{H}, \mathrm{OCH}_{3}\right)$, 3.94 (s, 3H, $\left.\mathrm{OCH}_{3}\right), 3.93$ (s, 3H, $\left.\mathrm{OCH}_{3}\right), 2.68\left(\mathrm{~s}, 3 \mathrm{H}, \mathrm{CH}_{3}\right)$, $2.42\left(\mathrm{~s}, 3 \mathrm{H}, \mathrm{CH}_{3}\right)$; IR (neat) $v: 2921\left(v_{\mathrm{Ar}-\mathrm{H}}\right), 1616\left(v_{\mathrm{C}=\mathrm{N}}\right)$,
1574 (Ar 骨架振动), 1506, 1431, 1297, $1223\left(v_{\mathrm{C}-\mathrm{N}}\right), 1133$, 993, 933, 851, 780, 657, $445 \mathrm{~cm}^{-1}$; $\mathrm{MS}\left(\mathrm{ESI}^{+}\right) \mathrm{m} / z(\%)$ : 452.00 ([M+1], 100). Anal. calcd for $\mathrm{C}_{22} \mathrm{H}_{25} \mathrm{~N}_{7} \mathrm{O}_{2} \mathrm{~S}: \mathrm{C}$ 58.52, H 5.58, N 21.71; found C 58.44, H 5.63, N 21.58.

4b: 黄绿色固体, $45.2 \%$, m.p. $188.4 \sim 189.6{ }^{\circ} \mathrm{C} ;{ }^{1} \mathrm{H}$ NMR $\left(\mathrm{CDCl}_{3}, 500 \mathrm{MHz}\right) \delta: 8.69(\mathrm{~s}, 1 \mathrm{H}), 8.48(\mathrm{~s}, 1 \mathrm{H})$, $7.73 \sim 7.70(\mathrm{~m}, 2 \mathrm{H}), 7.53 \sim 7.49(\mathrm{~m}, 2 \mathrm{H}), 7.27(\mathrm{~s}, 1 \mathrm{H})$, $7.16(\mathrm{~d}, J=9 \mathrm{~Hz}, 1 \mathrm{H}), 4.30(\mathrm{t}, J=5 \mathrm{~Hz}, 4 \mathrm{H}), 3.77(\mathrm{t}, J=5$ $\mathrm{Hz}, 4 \mathrm{H}), 4.04$ (s, 3H, $\left.\mathrm{OCH}_{3}\right), 4.01\left(\mathrm{~s}, 3 \mathrm{H}, \mathrm{OCH}_{3}\right), 2.70$ (s, $\left.3 \mathrm{H}, \mathrm{CH}_{3}\right) ;{ }^{13} \mathrm{C} \mathrm{NMR}\left(\mathrm{CDCl}_{3}\right) \delta: 180.7,172.4,164.5,158.6$, $154.8,152.3,148.9,132.1,129.6,129.2,128.9,128.8$, $117.4,110.6,108.9,103.2,56.5,56.4,48.7,48.6,21.2$; IR (neat) $v: 2851\left(v_{\mathrm{Ar}-\mathrm{H}}\right), 1616\left(v_{\mathrm{C}=\mathrm{N}}\right), 1572(\mathrm{Ar}$ 骨架振动), 1546, 1505, 1424, 1361, 1245, $1210\left(v_{\mathrm{C}-\mathrm{N}}\right), 1020,990$, 930, 759, 689, $565 \mathrm{~cm}^{-1}$; MS (ESI $\left.{ }^{+}\right) \mathrm{m} / \mathrm{z}(\%): 451.02$ $([\mathrm{M}+1], 100)$. Anal. calcd for $\mathrm{C}_{23} \mathrm{H}_{26} \mathrm{~N}_{6} \mathrm{O}_{2} \mathrm{~S}$ : C 61.31, $\mathrm{H}$ 5.82, N 18.65; found C 61.44, H 5.77, N 18.81 .

4c: 乳白色固体, $43.5 \%$, m.p. 221.2 221.9 ${ }^{\circ} \mathrm{C} ;{ }^{1} \mathrm{H}$ NMR (DMSO- $\left.d_{6}, 500 \mathrm{MHz}\right) \delta: 11.59(\mathrm{~s}, 1 \mathrm{H}), 8.54 \sim 8.50$ $(\mathrm{m}, 2 \mathrm{H}), 7.41(\mathrm{~d}, J=7 \mathrm{~Hz}, 1 \mathrm{H}), 7.28 \sim 7.22(\mathrm{~m}, 3 \mathrm{H})$, $6.90 \sim 6.89(\mathrm{~m}, 2 \mathrm{H}), 4.15(\mathrm{t}, J=5.0 \mathrm{~Hz}, 4 \mathrm{H}), 3.84(\mathrm{t}, J=$ $5.0 \mathrm{~Hz}, 4 \mathrm{H}), 3.93\left(\mathrm{~s}, 6 \mathrm{H}, \mathrm{OCH}_{3}, \mathrm{OCH}_{3}\right) ;{ }^{13} \mathrm{C} \mathrm{NMR}$ $\left(\mathrm{DMSO}-d_{6}\right) \delta: 181.3,162.7,157.6,154.7,149.1,148.5$, $131.8,130.7,120.0,119.9,117.4,116.6,110.7,108.1$, 107.2, 104.6, 56.4, 56.3, 48.6, 48.5; IR (neat) $v: 2920$ $\left(v_{\mathrm{Ar}-\mathrm{H}}\right), 1619\left(v_{\mathrm{C}=\mathrm{N}}\right), 1548(\mathrm{Ar}$ 骨架振动 $), 1503,1482$, 1242, $1213\left(v_{\mathrm{C}^{-} \mathrm{N}}\right), 1018,992,791,566 \mathrm{~cm}^{-1}$; $\mathrm{MS}\left(\mathrm{ESI}^{+}\right)$ $m / z \quad(\%): 453.07 \quad([\mathrm{M}+1], 100)$. Anal. calcd for $\mathrm{C}_{22} \mathrm{H}_{24} \mathrm{~N}_{6} \mathrm{O}_{3} \mathrm{~S}$ : C 58.39, H 5.35, N 18.57; found C 58.45, H 5.41, N 18.38 .

4d: 乳白色固体, 55.7\%, m.p. 220.2 221.3 ${ }^{\circ} \mathrm{C} ;{ }^{1} \mathrm{H}$ $\mathrm{NMR}\left(\mathrm{CDCl}_{3}, 500 \mathrm{MHz}\right) \delta: 8.70(\mathrm{~s}, 1 \mathrm{H}), 7.91(\mathrm{~s}, 1 \mathrm{H}), 7.29$ $(\mathrm{s}, 1 \mathrm{H}), 7.30 \sim 7.26(\mathrm{~m}, 2 \mathrm{H}), 7.19(\mathrm{~d}, J=2.5 \mathrm{~Hz}, 1 \mathrm{H}), 7.11$ (s, 1H), $6.94(\mathrm{~d}, J=9 \mathrm{~Hz}, 1 \mathrm{H}), 4.18(\mathrm{t}, J=5 \mathrm{~Hz}, 4 \mathrm{H}), 3.88$ (t, $J=5 \mathrm{~Hz}, 4 \mathrm{H}), 4.04(\mathrm{~s}, 3 \mathrm{H}), 4.01(\mathrm{~s}, 3 \mathrm{H}) ;{ }^{13} \mathrm{C}$ NMR $\left(\mathrm{CDCl}_{3}\right) \delta: 181.3,173.4,162.9,154.8,152.0,151.6,149.1$, 143.2 , 136.7, 126.7, 125.4, 119.3, 117.6, 110.6, 105.9, $103.2,56.5,56.5,48.7,48.8$; IR (neat) $v: 2922,1616$ $\left(v_{\mathrm{C}=\mathrm{N}}\right), 1578,1503,14751243,1213\left(v_{\mathrm{C}-\mathrm{N}}\right), 993,933,792$, 730, $564 \mathrm{~cm}^{-1}$; MS (ESI $\left.{ }^{+}\right) \mathrm{m} / z(\%): 487.03([\mathrm{M}+1], 100)$. Anal. calcd for $\mathrm{C}_{22} \mathrm{H}_{23} \mathrm{ClN}_{6} \mathrm{O}_{3} \mathrm{~S}$ : C 54.26, H 4.76, N 17.26; found C 54.41, H 4.72, N 17.19.

4e: 黄色固体, $23.5 \%$, m.p. $182.8 \sim 183.7{ }^{\circ} \mathrm{C} ;{ }^{1} \mathrm{H}$ $\operatorname{NMR}\left(\mathrm{CDCl}_{3}, 500 \mathrm{MHz}\right) \delta: 8.62(\mathrm{~s}, 1 \mathrm{H}), 8.03(\mathrm{~s}, 1 \mathrm{H}), 7.60$ (s, 1H), $7.27(\mathrm{~s}, 1 \mathrm{H}), 7.23(\mathrm{~s}, 1 \mathrm{H}), 7.16(\mathrm{~s}, 1 \mathrm{H}), 7.08$ (s, 
$1 \mathrm{H}), 4.19$ (t, $J=5 \mathrm{~Hz}, 4 \mathrm{H}), 3.90(\mathrm{t}, J=5 \mathrm{~Hz}, 4 \mathrm{H}), 4.00$ (s, $\left.3 \mathrm{H}, \mathrm{OCH}_{3}\right), 3.97\left(\mathrm{~s}, 3 \mathrm{H}, \mathrm{OCH}_{3}\right) ;{ }^{13} \mathrm{C} \mathrm{NMR}\left(\mathrm{CDCl}_{3}\right) \delta$ : $180.7,172.3,162.9,155.0,152.3,148.9,144.5,144.2$, $136.8,119.6,116.1,112.4,110.7,110.5,103.5,102.4$, 56.6, 56.5, 48.9, 48.6; IR (neat) v: 2920, $1616\left(v_{\mathrm{C}=\mathrm{N}}\right)$, 1575, 1504, 1476, 1425, $1210\left(v_{\mathrm{C}^{-} \mathrm{N}}\right), 993,933,561 \mathrm{~cm}^{-1}$; MS (ESI $\left.{ }^{+}\right) m / z(\%): 610.91([\mathrm{M}+1], 100)$. Anal. calcd for $\mathrm{C}_{22} \mathrm{H}_{22} \mathrm{Br}_{2} \mathrm{~N}_{6} \mathrm{O}_{3} \mathrm{~S}$ : C 43.29, $\mathrm{H}$ 3.63, N 13.77; found $\mathrm{C}$ 43.20, H 3.66, N 13.65 .

4f: 乳白色固体, $32.4 \%$, m.p. $194.5 \sim 195.4{ }^{\circ} \mathrm{C} ;{ }^{1} \mathrm{H}$ NMR $\left(\mathrm{CD}_{3} \mathrm{COCD}_{3}, 500 \mathrm{MHz}\right) \delta: 12.01(\mathrm{~s}, 1 \mathrm{H}), 10.65(\mathrm{~s}$, $1 \mathrm{H}), 8.56(\mathrm{~s}, 1 \mathrm{H}), 8.45$ (s, 1H), 7.39 (d, $J=2 \mathrm{~Hz}, 1 \mathrm{H}), 7.31$ $(\mathrm{s}, 1 \mathrm{H}), 7.22(\mathrm{~s}, 1 \mathrm{H}), 7.12(\mathrm{~s}, 1 \mathrm{H}), 4.26(\mathrm{t}, J=5 \mathrm{~Hz}, 4 \mathrm{H})$, 3.89 (t, $J=5 \mathrm{~Hz}, 4 \mathrm{H}), 3.99\left(\mathrm{~s}, 3 \mathrm{H}, \mathrm{OCH}_{3}\right), 3.98(\mathrm{~s}, 3 \mathrm{H}$, $\left.\mathrm{OCH}_{3}\right), 1.46\left[\mathrm{~s}, 9 \mathrm{H}, \mathrm{C}\left(\mathrm{CH}_{3}\right)_{3}\right], 1.29\left[\mathrm{~s}, 9 \mathrm{H}, \mathrm{C}\left(\mathrm{CH}_{3}\right)_{3}\right] ;{ }^{13} \mathrm{C}$ NMR $\left(\mathrm{CD}_{3} \mathrm{COCD}_{3}\right) \delta: 180.6,163.1,154.9,153.2,151.9$, $149.4,148.9,140.5,136.2,126.0,125.4,124.9,117.5$, 111.1, 106.8, 103.7, 55.4, 55.4, 48.6, 48.0, 34.8, 33.8, 31.1, 30.7; IR (neat) v: 2957, $1617\left(v_{\mathrm{C}=\mathrm{N}}\right), 1574,1505,1478$, 1425, 1307, $1209\left(v_{\mathrm{C}^{-} \mathrm{N}}\right), 1020,993,937,849,560 \mathrm{~cm}^{-1}$; MS (ESI $\left.{ }^{+}\right) m / z(\%): 565.11([\mathrm{M}+1], 100)$. Anal. calcd for $\mathrm{C}_{30} \mathrm{H}_{40} \mathrm{~N}_{6} \mathrm{O}_{3} \mathrm{~S}$ : C 63.80, H 7.14, N 14.88; found C 63.95, $\mathrm{H}$ 7.08, N 14.80.

\section{6 体外抗肿瘤活性测试}

用 MTT(四氮唑蓝) 比色法测试了化合物的体外抗 肿瘤活性. 实验所用的三种肿瘤细胞株分别为人胃癌细 胞(SGC-7901)、人口腔表皮样癌细胞(KB)和人纤维肉瘤 细胞(HT-1080). 将药物配制成以完全培养基为溶剂, 阿霉素 $(\mathrm{ADM})$ 作为对照药剂. 将生长至对数生长期的细 胞消化、离心, 制成细胞悬液, 吹匀并将其密度调至 4 $6 \times 10^{4}$ 个 $/ \mathrm{mL}$, 以 $180 \mu \mathrm{L} /$ 孔接种到 96 孔板中, 将 96 孔 板置于 $37{ }^{\circ} \mathrm{C}$ 、含 $5 \%$ 的 $\mathrm{CO}_{2}$ 、饱和湿度的培养箱中孵育 $24 \mathrm{~h}$. 每种化合物分为 4 个浓度梯度, 其浓度分别为 0.1 , 1.0, 10 和 $100 \mu \mathrm{mol} / \mathrm{L}$. 取各个剂量的药液加入 96 孔板 中, 每孔 $20 \mu \mathrm{L}$, 每剂量 3 个复孔. 加药完毕放入培养箱 中孵育 $72 \mathrm{~h}$. 然后加入配置好的 MTT 溶液, 每孔 $20 \mu \mathrm{L}$, 放入培养箱中继续孵育 $4 \mathrm{~h}$. 取出孵育 $4 \mathrm{~h}$ 的 96 孔板, 将 其中的 MTT 溶液轻轻甩出, 每孔加 $100 \mu \mathrm{L}$ 的 DMSO, 微量振荡仪上振荡 $5 \mathrm{~min}$ 以使甲瓒完全溶解, 用酶标仪 在 $492 \mathrm{~nm}$ 下测光密度(OD)值, 并记录数据.

按下列公式计算药物对肿瘤细胞体外增殖的抑制 率(Inhibition Rate, IR):

$$
\mathrm{IR}=\frac{\overline{\mathrm{OD}}_{\text {control }}-\overline{\mathrm{OD}}_{\text {sample }}}{\overline{\mathrm{OD}}_{\text {control }}-\overline{\mathrm{OD}}_{\text {blank }}} \times 100 \%
$$

$\overline{\mathrm{OD}}_{\text {control }}$ 未加药孔的 $\mathrm{OD}$ 值, $\overline{\mathrm{OD}}_{\text {sample }}$ 加药孔的 $\mathrm{OD}$ 值, $\overline{\mathrm{OD}}_{\text {blank }}$ 为只加 DMSO 孔的 OD 值.

\section{2 结果与讨论}

\subsection{6,7-二甲氧基-4-哌嗪喹唑啉缩氨基硫艮化合物 $4 a$ 的结构}

该反应实际上是 6,7-二甲氧基-4-哌嗪基喹唑啉与 2-乙酰基吡啶肼基二硫代甲酸甲酯发生的缩合反应(脱 去一分子甲硫醇). 根据 2-乙酰基吡啶肼基二硫代甲酸 甲酯的结构可知，其应具有顺反异构体，而其 ${ }^{1} \mathrm{H}$ NMR 谱图可以验证这一点. 所以，从理论上来讲，目标化合 物 $4 \mathbf{a}$ 也应有顺反异构情况. 分析 $\mathbf{4 a}$ 的 ${ }^{1} \mathrm{H} N \mathrm{NM}$ 谱图发 现, 在 $\delta 4.43,3.94,4.26$ 和 3.75 处有四个三重峰, 其积分 之比约为 $4 / 4 / 4 / 4$, 这四个峰应该是两种哌嗪环上四个亚 甲基的氢; 在 $\delta 3.96,3.94,3.94$ 和 3.93 处有四个单峰，其 积分比约为 $3 / 3 / 3 / 3$, 这四个单峰应该是两种喹唑啉环上 四个甲氧基的氢; 在 $\delta 2.68$ 和 2.42 处有两个单峰，其积 分比约为 $3 / 3$, 应该是亚胺中碳上所连甲基的氢; $\mathbf{4 a}$ 的 $Z$ 式存在着 $\mathrm{N} \cdots \mathrm{H}-\mathrm{N}$ 氢键, 所以受氢键的影响 $\mathrm{N}$ 上氢的 化学位移应出现在低场, 恰恰谱图的低场 $\delta 14.83$ 处有 一个小的宽峰. 据此, 推断化合物 $\mathbf{4 a}$ 的两种异构体如图 2 所示.

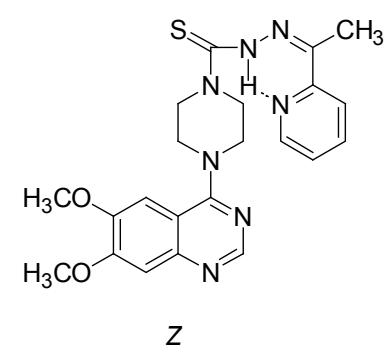

图 2 化合物 $\mathbf{4 a}$ 的两种异构体

\section{2 体外抗肿瘤活性测定}

由于缩氨基硫脲和喹唑啉化合物具有细胞毒性 ${ }^{[16]}$, 我们采用常规的 MTT 比色实验，测试了所合成化合物 4 对人胃癌细胞(SGC-7901)、人口腔表皮样癌细胞(KB) 和人纤维肉瘤细胞(HT-1080)的体外抗肿瘤活性, 实验 结果见表 1. 比较化合物 $\mathbf{4}$ 的结构可知, 化合物 $\mathbf{4 a}$ 和 $\mathbf{4 b}$ 有相同 $\mathrm{R}^{\prime}$ (甲基)，而 $\mathrm{R}$ 分别为 2-吡啶基和苯基； $4 \mathbf{c} \sim \mathbf{4 f}$ 的 $\mathrm{R}$ 部分相似，均为取代 2-羟基苯基, $\mathrm{R}^{\prime}$ 部分均为氢.

从表 1 中可见，所合成的化合物对 3 种人肿瘤细胞 的体外生长显示不同的抑制作用. 对于人胃癌细胞 (SGC-7901)，化合物 $\mathbf{4 a}$ 显示出一定的抑制活性，其 $\mathrm{IC}_{50}$ 
值为 $45.72 \mu \mathrm{mol} \cdot \mathrm{L}^{-1}$, 化合物 $\mathbf{4 c}$ 和 $\mathbf{4 f}$ 的抑制活性低于 $4 \mathbf{a}$, 而化合物 $4 \mathrm{~b}, 4 \mathrm{~d}$ 和 $4 \mathrm{e}$ 在最大浓度 $\left(10^{2} \mu \mathrm{mol} \cdot \mathrm{L}^{-1}\right)$ 时, SGC-7901 细胞的抑制率未达到 50\%. 对于人口腔表皮 样癌细胞 $(\mathrm{KB})$, 化合物 $\mathbf{4 a}, \mathbf{4 b}, \mathbf{4 c}$ 和 $\mathbf{4 f}$ 的 $\mathrm{IC}_{50}$ 值均在 $\left(10^{2}\right.$ $\left.\mu \mathrm{mol} \cdot \mathrm{L}^{-1}\right)$, 而化合物 $\mathbf{4 d}$ 和 $\mathbf{4 e}$ 的抑制活性更弱. 从表 1 中可以清晰地看到, 化合物 $\mathbf{4 a} \sim \mathbf{4 f}$ 对人纤维肉瘤细胞 (HT-1080) 的抑制作用明显强于对人胃癌细胞 (SGC-7901)和人口腔表皮样癌细胞(KB)的抑制作用. 比 较化合物 $\mathbf{4 a} \sim \mathbf{4 f}$ 对 $\mathrm{HT}$ 细胞的 $\mathrm{IC}_{50}$ 值, 可以看出缩氨基 硫脲部分连有不同的结构对化合物的生物活性有很大 影响. 其中, 连有吡啶的 $\mathbf{4 a}$ 对 HT 细胞表现出较好的抑 制活性, 其 $\mathrm{IC}_{50}$ 值为 $10.43 \mu \mathrm{mol} / \mathrm{L}$; 连有大的取代基一 叔丁基的化合物 $4 \mathbf{f}$ 对 HT 细胞也表现出较好的抑制活性 $\left(\mathrm{IC}_{50}\right.$ 值为 $\left.28.57 \mu \mathrm{mol} / \mathrm{L}\right)$, 但这两种化合物的抑制活性还 远低于对照药 ADM, 其结构还有待于进一步优化.

表 1 化合物 4a 4f 对 SGC-7901, KB 和 HT1080 细胞的 $\mathrm{IC}_{50}$ 值 ${ }^{a}$

Table $1 \mathrm{IC}_{50}$ values of $\mathbf{4 a} \sim \mathbf{4 f}$ for SGC-7901, KB and HT-1080 cell lines

\begin{tabular}{|c|c|c|c|c|c|}
\hline \multirow{2}{*}{ Compd } & \multirow{2}{*}{$\mathrm{R}$} & \multirow{2}{*}{$\mathrm{R}^{\prime}$} & \multicolumn{3}{|c|}{$\mathrm{IC}_{50}\left(\mu \mathrm{mol} \cdot \mathrm{L}^{-1}\right)$} \\
\hline & & & SGC7901 & $\mathrm{KB}$ & HT1080 \\
\hline $4 a$ & & $\mathrm{CH}_{3}$ & 45.72 & 158.75 & 10.43 \\
\hline $4 b$ & & $\mathrm{CH}_{3}$ & - & 465.67 & 194.83 \\
\hline $4 c$ & & $\mathrm{H}$ & 204.53 & 151.92 & 35.35 \\
\hline $4 d$ & & $\mathrm{H}$ & - & - & 59.30 \\
\hline $4 e$ & $\mathrm{Br}$ & $\mathrm{H}$ & - & - & 176.67 \\
\hline $4 f$ & & $\mathrm{H}$ & 205.73 & 299.81 & 28.57 \\
\hline ADM & & & 0.256 & 0.498 & 0.017 \\
\hline
\end{tabular}

a“一”指化合物的最大浓度时的抑制率未达到 $50 \%$.

综上所述, 我们设计、合成了一类新型的含有喹唑 啉和缩氨基硫脲结构的化合物. 初步的体外抗肿瘤活性 测试结果表明, 化合物 $4 \mathbf{a}$ 和 $4 \mathrm{f}$ 对人纤维肉瘤细胞 (HT-1080)的体外生长具有明显的抑制作用. 下一步的
工作将集中于通过类似物的设计、合成及构效关系研究， 以发现更强的抗肿瘤活性的化合物.

致谢 活性篎选试验由沈阳药科大学药理实验室测定, 在此对其给予的帮助表示诚挚的谢意.

\section{References}

1 Lim, J. K.; Negash, K.; Hanraham, S. M.; VanBrocklin, H. F. J. Labelled Compd. Radiopharm. 2000, 43, 1183.

2 Nakamura, H.; Onagi, S. Tetrahedron Lett. 2006, 47, 2539.

3 Fang, H.; Li, M. Y.; Xia, L. Chin. Chem. Lett. 2007, 18, 41.

4 Lokker, N. A.; Sullivan, C. M.; Hollenbach, S. J.; Israel, M. A.; Giese, N. A. Cancer Res. 2002, 62, 3729.

5 Nakamura, K.; Yamamoto, A.; Kamishohara, M.; Takahashi, K.; Tagushi, K.; Taguchi, E.; Miura, T.; Kubo, K.; Shibuya, M.; Isoe, T. Mol. Cancer Therapeutics 2004, 3, 1639.

6 Ding, M.-W.; Yang, S.-J.; Chen, Y.-F. Chin. J. Org. Chem. 2004, 24, 923 (in Chinese).

(丁明武, 杨尚君, 陈云峰, 有机化学, 2004, 24, 923.)

7 Yang, S.; Liu G.; Song, B.-A.; Jin, L.-H.; Hu, D.-Y.; Zhang, S.-M. Chin. J. Org. Chem. 2006, 26, 1429 (in Chinese). (杨松, 刘刚, 宋宝安, 金林红, 胡德禹, 张素梅, 有机化 学, 2006, 26, 1429.)

8 Yong, J.-P.; HaJi, A.-A. Chin. J. Org. Chem. 2008, 28, 1204 (in Chinese).

(雍建平，阿吉艾克拜尔・艾萨，有机化学，2008，28， 1204.)

9 Li, M.-X.; Zhang, D.; Zhang, L.-Z.; Niu, J.-Y.; Ji, B.-S. J. Org. Chem. 2011, 696, 852.

10 Duan, L.-P.; Zhang, H.-B. Arabian J. Chem. 2011, 4, 231.

11 Bharti, N.; Maury, M. R.; Naqvi, F.; Azam, A. Bioorg. Med. Chem. Lett. 2000, 10, 2243.

12 Fujii, N.; Mallari, J. P.; Hansell, E. J.; Mackey, Z.; Doyle, P.; Zhou, Y. M.; Gut, J.; Rosenthal, P. J.; McKerrow, J. H.; Guy, R. K. Bioorg. Med. Chem. Lett. 2005, 15, 121.

13 Easmon, J.; Pürstinger G.; Heinisch G.; Roht, T.; Fiebig H. H.; Holzer, W.; Jäger, W.; Jenny, M.; Hofmann, J. J. Med. Chem. 2001, 449, 2164.

14 Li, Q. H. J. Southwest Univ. Nat. (Nat. Sci. Ed.) 2009, 35, 309 (in Chinese).

(李清寒，西南民族大学学报(自然科学版), 2009, 35, 309.)

15 Hu, W.-X.; Sun, N.; Yang, Z.-Y. Chin. J. Med. Chem. 2001, 11, 129 (in Chinese).

(胡惟孝, 孙楠, 杨忠愚, 中国药物化学杂志, 2001, 11, 129.)

16 Aly, M. M.; Mohamed, Y. A.; El-Bayouki, K. A. M.; Basyouni, W. M.; Abbas, S. Y. Eur. J. Med. Chem. 2010, 45, 3365 . 